\title{
A NOTE ON THE LICKORISH-MILLETT-TURAEV FORMULA FOR THE KAUFFMAN POLYNOMIAL
}

\author{
JÓZEF H. PRZYTYCKI
}

(Communicated by Ronald Stern)

\begin{abstract}
We use the idea of expressing a nonoriented link as a sum of all oriented links corresponding to the link to present a short proof of the LickorishMillett-Turaev formula for the Kauffman polynomial at $z=-a-a^{-1}$. Our approach explains the observation made by Lickorish and Millett that the formula is the generating function for the linking number of a sublink of the given link with its complementary sublink.
\end{abstract}

We have conjectured [7] and partially proved (in April 1986) that the Kauffman polynomial of a knot at $z=-a-a^{-1}$ is equal to one. Lickorish and Millett solved the conjecture (in August 1986) and found the formula for the Kauffman polynomial at $z=-a-a^{-1}$ for any link [5] (see also [6]). The formula was also independently discovered, in a more general context, by Turaev [8]. We give here the "calculationfree" approach to the formula using the idea of presenting a nonoriented link as a sum of all corresponding oriented links.

To fix the notation we recall the standard definition of the Kauffman polynomial, $F_{L}(a, z)$, of unoriented framed links in $S^{3}: F_{L}(a, z) \in Z\left[a^{ \pm 1}, z^{ \pm 1}\right]$ is uniquely determined by the following properties:

(1) $F_{T_{1}}=1$, where $T_{1}$ is the unknot with 0 -framing.

(2) $F_{L^{(1)}}=a F_{L}$, where $L^{(1)}$ is the framed link obtained from $L$ by adding a full right-hand twist to the framing of $L$.

(3) $F_{L_{+}}+F_{L_{-}}=z\left(F_{L_{0}}+F_{L_{\infty}}\right)$, where $L_{+}, L_{-}, L_{0}$, and $L_{\infty}$ denote four unoriented links which are the same, except in a small ball where they look as in Figure 1 on the next page, and the framing, in the formula, is assumed to be vertical to the plane of the projection.

The Kauffman polynomial of oriented links, $F_{L}(a, z)$, which is an invariant of ambient isotopy, is defined as the Kauffman polynomial of the corresponding unoriented 0 -framed link (i.e., the framing is given by the Seifert surface of the oriented link). The Kauffman polynomial of an unoriented diagram $D$, which is an invariant of regular isotopy, is equal to the Kauffman polynomial of the link given by the diagram with the vertical framing.

We will derive the formula (of Lickorish, Millett, and Turaev) from the idea of presenting an unoriented link as a sum of oriented links corresponding to it (the formula is obtained without guessing it in advance).

Received by the editors January 5, 1993.

1991 Mathematics Subject Classification. Primary 57M25. 

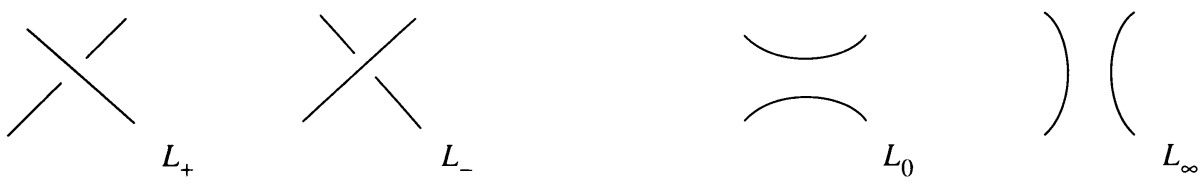

FIGURE 1

Definition 1. Let $\mathscr{L}_{\text {fr }}$ denote the set of ambient isotopy classes of framed unoriented links in $S^{3}$ and $\overrightarrow{\mathscr{L}}_{\mathrm{fr}}$ the set of ambient isotopy classes of framed oriented links in $S^{3}$. Further, let $R=Z\left[a^{ \pm 1}\right]$ and $R X$ denote the free $R$-module with basis $X$. We define a "transfer" map $\tau: R \mathscr{L}_{\mathrm{fr}} \rightarrow R \overrightarrow{\mathscr{L}}_{\mathrm{fr}}$ by $\tau(L)=\sum_{L^{\prime} \in \mathrm{OR}(L)} L^{\prime}$, where $\operatorname{OR}(L)$ is the set of all orientations of $L$. For example, $\tau(O)=0+0$.

Now consider the following very simple invariant of framed oriented links.

Definition 2. $g: R \overrightarrow{\mathscr{L}}_{\mathrm{fr}} \rightarrow Z\left[a^{ \pm 1}\right]$ is defined by $g(L)=(-1)^{\operatorname{com}(L)} a^{\mathrm{fr}(L)}$, where $\operatorname{com}(L)$ is the number of components of $L$ and $\operatorname{fr}(L)$ is the framing number of $L$; that is, the number of right-hand twists that must be removed from the framing of $L$ to reach the 0 -framing.

We will analyze the composition $g \tau$ and first show that it is the Kauffman polynomial at $z=-a-a^{-1}$ multiplied by -2 . Then we will easily evaluate $F\left(a,-a-a^{-1}\right)$ using its relation to $g \tau$.

Lemma 3. (a) $g \tau\left(L_{+}\right)+g \tau\left(L_{-}\right)=-\left(a+a^{-1}\right)\left(g \tau\left(L_{0}\right)+g \tau\left(L_{\infty}\right)\right)$.

(b) $g \tau(L)=-2 F_{L}\left(a,-a-a^{-1}\right)$, where $L$ is any unoriented framed link.

Proof. We must consider two cases:

(1) (the case of a self-crossing) In this case $L_{0}$ or $L_{\infty}$ (say $L_{0}$ ) has one more component than the other three links involved in the relation. So $L_{0}$ has twice as many orientations as the other links. Half of these orientations are the same as in $L_{\infty}$, and the other half agrees with that of $L_{+}$. The formula in Lemma 3(a) follows easily when one observes that if an oriented diagram $D$ has the vertical framing then $\operatorname{fr}(D)=\operatorname{Tait}(D)$, where $\operatorname{Tait}(D)$ is the algebraic sum of the signs of the crossings of $D$.

(2) (the case of a mixed crossing) In this case $L_{+}$(and $L_{-}$) has one more component than $L_{0}$ (and $L_{\infty}$ ) and therefore twice as many possible orientations. As in (1) formula (a) follows almost immediately.

(b) The formula in (a) agrees with that for the Kauffman polynomial $F_{L}\left(a,-a-a^{-1}\right)$. Furthermore in both cases, if the framing of a link is changed by adding to it a positive twist then the invariant is multiplied by $a$. Therefore, we must compare our two invariants for the 0 -framed unknot, $T_{1}$. Then $g \tau\left(T_{1}\right)=-2$, and $F_{T_{1}}\left(a,-a-a^{-1}\right)=1$. Lemma 3(b) follows.

Lemma 4. Let $L$ be an oriented link in $S^{3}$ with 0 -framing and $p(L)$ the same framed link but without an orientation. Then

$$
g \tau p(L)=(-1)^{\operatorname{com}(L)} \sum_{S \subset L} a^{-41 \mathbf{k}(S, L-S)}
$$

where the summation is taken over all sublinks $S$ of $L$ (including $S=\varnothing$ ) and $1 \mathrm{k}(S, L-S)$ denotes the global linking number between $S$ and $L-S$. 
Proof. If $S$ is a sublink of $L$ and $L_{S}$ is the oriented framed link obtained from $L$ by changing the orientation of the components in $S$ but keeping the framing of $L$ then $\operatorname{fr}\left(L_{S}\right)=-4 \operatorname{lk}(S, L-S)$; in particular $\operatorname{fr}\left(L_{\varnothing}\right)=0$. From the above observation the formula for $g \tau p(F)=g\left(\sum_{S \subset L} L_{S}\right)$ follows immediately.

As a corollary we have the Lickorish-Millett-Turaev formula.

\section{Theorem 5.}

$$
F_{L}\left(a,-\left(a+a^{-1}\right)\right)=\left((-1)^{\operatorname{com}(L)-1} / 2\right) \sum_{S \subset L} a^{-4 \mathrm{l}(S, L-S)} .
$$

Proof. We combine Lemmas 3 and 4.

The idea used in the note was applied for the first time in [1] and ascribed to Dennis Johnson (see also [9, 2]). There are other applications of the idea in the theory of skein modules [3], which we hope to describe in the future.

\section{REFERENCES}

1. W. Goldman, Invariant functions of Lie groups and Hamiltonian flows of surface group representation, Invent. Math. 85 (1986), 263-302.

2. J. Hoste and J. H. Przytycki, Homotopy skein modules of orientable 3-manifolds, Math. Proc. Cambridge Philos. Soc. 108 (1990), 475-488.

3. _ A survey of skein modules of 3-manifolds, "Knots 90", De Gruyter, Berlin, New York, 1992, pp. 363-379.

4. L. H. Kauffman, On knots, Ann. of Math. Stud., vol. 115, Princeton Univ. Press, Princeton, NJ, 1987.

5. W. B. R. Lickorish and K. C. Millett, An evaluation of the F-polynomial of a link, Differential Topology, Proc. 2nd Topology Symp. (Siegen/FRG 1987), Lecture Notes in Math., vol. 1350, Springer-Verlag, New York, 1988, pp. 104-108.

6. A. S. Lipson, Some more states models for link invariants, Pacific J. Math. 152 (1992), 337-346.

7. H. R. Morton, Problems, Braids (J. S. Birman and A. Libgober, eds.), Contemp. Math., vol. 78, Amer. Math. Soc., Providence, RI, 1988, pp. 557-574.

8. V. G. Turaev, The Yang-Baxter equation and invariants of links, Invent. Math. 92 (1988), 527-553.

9. $\ldots$ Skein quantization of Poisson algebras of loops on surfaces, Ann. Sci. École Norm. Sup. (4) 4 (1991), 635-704.

Department of Mathematics and Computer Science, Odense University, Campusvej 55, DK-5230, Odense M, Denmark

E-mail address: Jozef@imada.ou.dk 